\title{
Von Brunn's Nests in the Ureter of Two Cats
}

\author{
Gianfranco Militerno ${ }^{1 *}$, Patrizia Bassi ${ }^{1}$, Giuliano Bettini' ${ }^{1}$, Roberto Nannini ${ }^{2}$ \\ ${ }^{1}$ Department of Veterinary Medical Sciences, University of Bologna, Bologna, Italy \\ ${ }^{2}$ Complex Operative Unit of Pathology, Imola (Bologna), Italy \\ Email: * gia nfranco.militerno@unibo.it
}

How to cite this paper: Militerno, G., Bassi, P., Bettini, G. and Nannini, R. (2017) Von Brunn's Nests in the Ureter of Two Cats. Open Journal of Veterinary Medicine, 7, 162-167.

https://doi.org/10.4236/ojvm.2017.711017

Received: October 19, 2017

Accepted: November 21, 2017

Published: November 24, 2017

Copyright $\odot 2017$ by authors and Scientific Research Publishing Inc. This work is licensed under the Creative Commons Attribution International License (CC BY 4.0).

http://creativecommons.org/licenses/by/4.0/

\begin{abstract}
The aim of this work is to emphasize the importance of a differential diagnosis of von Brunn's nests in cats from other urothelial neoplastic disorders, as transitional cell carcinoma, for a subsequent optimal care. Von Brunn's nests and cysts are submucosal benign urothelial processes, related to irritative stimuli (calculi and urinary infections), characterized by an invagination with buds and clusters of normal urothelium in the lamina propria. They are common findings in the urinary bladder of human beings, but few cases have been described in dogs and cats. We report macroscopic, histopathological and, in one case, immunohistochemical features of these rare forms in the left ureters of two cats. Macroscopic evidence during surgery of ureteral nodules in two cats was accompanied by histopathological diagnosis and, in one case, by immunohistochemical assessment. Histopathology was coherent with a rare condition characterized by nests and islands of normal urothelium in the lamina propria and submucosa, with formation of cysts and moderate focal subacute inflammation. In one case epithelial cells of the nests showed well differentiated urothelial cells with an intense immunoreactivity to pan-cytokeratin (CK AE1/AE3), CK19 in the first outer layers, a slight immunoreactivity to CK20 and a low proliferative activity using MIB-1 (Ki67).
\end{abstract}

\section{Keywords}

Von Brunn's Nests, Urothelium, Ureter, Neoplasms, Histopathology, Cat

\section{Introduction}

In the urinary tract, von Brunn's nests and cysts are wall thickening or nodules caused by the action of irritative stimuli (bacterial infections, urolithiasis and other causes), characterized by an invagination of clusters of normal urothelium in the lamina propria [1] [2] [3] [4]. These lesions are submucosal benign urothelial processes and they are common findings in the urinary bladder of human 
beings. They are reported in $89 \%$ of normal human urinary bladders [5]. Very few cases have been described in dogs and cats, but no one in ureters [6] [7]. Brunn's nests can also occur in renal pelvis [8]. We report macroscopic, histopathological and, in one case, immunohistochemical features of these rare forms found in the left ureters of two cats, after ureterectomy due to urinary obstruction with slight monolateral hydronephrosis.

\section{Case Report}

Surgical samples of left ureter of a 5-year-old, European, sterilized female cat (case 1) and a 6-year-old, European, neutered male cat (case 2) were sent to the Pathological Anatomy Laboratory Service of the Department of Veterinary Medical Sciences of Bologna for diagnosis. Histological sections of the second case, stained with haematoxylin and eosin ( $\mathrm{H} \& \mathrm{E})$ were sent for consulting and diagnosis. Case 1 and case 2 were sent from two different veterinarians. Both cases were accompanied by a medical history of haematuria and urinary obstruction with slight monolateral hydronephrosis, discovered with ultrasonographic investigation. Clinical and ultrasonographic examinations revealed nodular ureteral lesions. Histopathological examination was carried out after ureterectomy of the affected sites of both cats.

In both cases, macroscopical findings during surgery were coherent with an isolated, nodular, and compact mass of about $2 \mathrm{~cm}$ diameter at $1 \mathrm{~cm}$ from the bladder.

\section{Material and Methods}

In case 1 , tissue samples of the ureteral lesions were obtained, fixed in $10 \%$ buffered formalin, embedded in paraffin wax, sectioned at $4 \mu \mathrm{m}$ and stained with $\mathrm{H}$ \& E for histopathological examination. To better define the characteristics of von Brunn's nests and its urothelium, unstained paraffin-embedded sections were processed using the streptavidin-biotin peroxidase technique and the immunohistochemical evaluation of pan-cytokeratin (PanCK, CK AE1/AE3 dilution 1:300, Dako-Glostrup, Denmark), CK19 (dilution 1:400, Histo-line Lab., Milan, Italy), CK20 (dilution 1:400, Dako), and MIB-1 (dilution 1:600, Dako).

Immunohistochemical sections of human ureter and urinary bladder samples were used as control cases for immunohistochemical evaluation of CK20.

Histological sections of the second case were immediately observed.

A normal ureter from a 5-year-old European, female cat, examined during a necropsy, has been sampled as case control for histology of case 1 and case 2 .

\section{Results}

Microscopic examination showed considerable thickening of the muscular wall due to multifocal fibrosis, multifocal lacking of the lining epithelium in transverse sections of the ureteral lumen, and foci of urothelial islands of various sizes in the lamina propria without any cytological atypia or mitosis and with occa- 
sional central cystic cavities (Figure 1(A) and Figure 1(B)). In case 1, multifocal areas of oedema, hyperemia/hemorrhage, angiogenesis and mild to moderate widespread lymphoplasmacytic infiltrate were also present. The cystic cavities contained macrophages full of lipofuscin, hemosiderin, erythrocytes and eosinophilic amorphous material. Urothelial islands showed intense and diffuse immunoreactivity for pan-cytokeratin (CK AE1/AE3). In the first outer layers the islands were positive for CK19 (Figure 2(A)), and slightly positive for CK20 in well differentiated umbrella cells of the superficial layer (Figure 2(B)). MIB-1 immunoreactivity was observed in a low percentage of basal urothelial cells (less than $10 \%$ ), reaching a positivity rate of proliferating cells similar to that observed in benign tumors or in low-grade transitional cell carcinomas. Multifocal fibrosis, characterized by activated fibroblasts and immature connective tissue, was observed. Foci of hemorrhages, lymphocytes, plasma-cells and macrophages were present also in adventitia and peripheral adipose tissue. In this case, diagnosis was firstly coherent with a chronic lymphoplasmacytic transmural ureteritis, with oedematous and hemorrhagic foci of acute flogosis. Inflammation was poor in the other case, where urothelial nests in the lamina propria appeared multifocally dilated with focal areas of mucous metaplasia (Figure 1(B)). In both cases, these findings and the anamnesis of chronic obstructive urinary disease allowed to hypothesize that the presence of epithelial islands was coherent with the hyperplastic condition, better known as von Brunn's nests and cysts.

\section{Discussion}

These two cases showed Brunn's nests in the left ureters of two cats with two histological patterns. In the first case, islands of transitional epithelium were seen inside the ureteral wall with normal ureter, surrounded by fibrous tissue and foci of hemorrhage and lymphoplasmacytic inflammation. These findings suggested initially a chronic ureteritis probably caused by an unspecified bacterial infection. In fact, medical history did not refer of any other disorders, as urolithiasis. Disposition of epithelial nests constituted by well differentiated cells were also coherent with a hyperplastic/neoplastic condition. The second case was only microscopically examined: no samples but histological sections were sent for diagnosis. In this case, benign proliferative epithelial nests were characterized by multifocal cysts and a low number of compact nests with a poor inflammatory reaction. In this case differential diagnosis included an adenoma with cystic component. No other sections or immunohistochemical assessment were available due to lack of formalin fixed samples or paraffin blocks. It is important to know the histological features of von Brunn's nests, since it is not always easy to distinguish these islands from a papilloma or a well-differentiated urothelial carcinoma with endophytic (inverted) growth patterns. This is mostly true in differential diagnosis of neoplastic disorders of the urinary bladder. In fact, primitive benign or malignant tumors of the transitional epithelium represent a very rare finding in the ureters and in renal pelvis both in domestic 


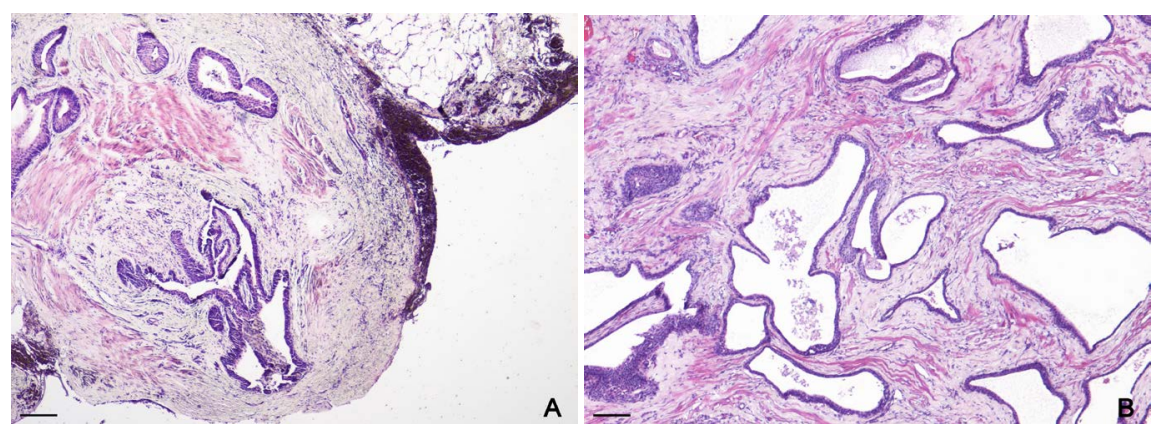

Figure 1. (A) case 1 and (B) case 2: cats, ureter, histological images of von Brunn's nests and cysts. In (A) are also visible a cross section of the ureter, lower left of the image, focal lymphocitic infiltrates and hemorrhage on the right. In (B) urothelial islands (von Brunn's nests) are multifocally dilated. $\mathrm{H} \& \mathrm{E}, \mathrm{Bar}=200 \mu \mathrm{m}$.

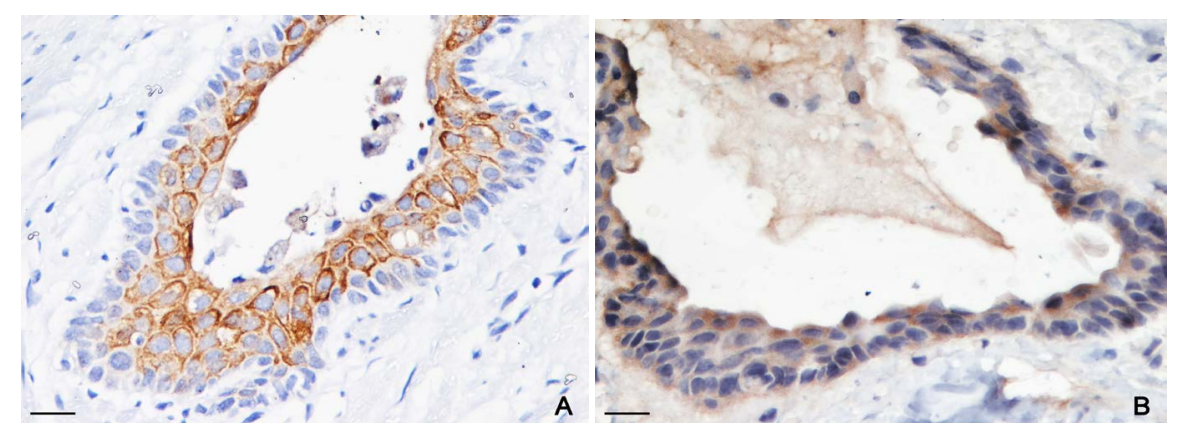

Figure 2. Case 1: cat, ureter, immunoistochemical images of von Brunn's nests. (A), first outer layers of the islands positive for CK19; (B), well differentiated umbrella cells of the superficial layer slightly positive for CK20. Haematoxylin counterstain, Bar $=25 \mu \mathrm{m}$.

animals and in human beings, as they generally arise elsewhere in the urinary tract, especially in the bladder. Nodules or plaques in the ureters could be the result of a synchronous or metachronous spread with implantation of malignant cells by primitive tumors from other sites of the urinary system [4] [8]. Von Brunn's nests are transitional cell groups in the lamina propria which appear attached or detached from the urothelium and when they seem to be relatively deep and isolated within the lamina propria, the diagnosis can be very difficult. It is also possible that isolated nests and buds are the result of a tangential cutting of hyperplastic urinary epithelium [8]. Therefore, von Brunn's nests and cysts can occur at every level of urothelium, stimulated by inflammatory responses with hyperplasia due to urolithiasis, excreted metabolites, bacterial infections or traumatic injuries from urinary catheterization. In dogs and humans, von Brunn's nests, cystitis cystica and cystitis glandularis are hyperplastic responses reported in cases of chronic urinary bladder irritation [1] [2] [3] [4]. Biopsy is not necessary and the histological examination after the total removal is more useful. The diagnosis should be made with caution and care during macroscopic intraoperative examination. It is important to see foci of metastatic spread of a presumptive urothelial carcinoma and subsequently to evaluate histologically the presence of atypia, the number of mitoses, the presence of an invasive growth pattern, the disposition of the cells, the urothelial layers, normally 
not more than 3 - 6. Immunohistochemical assessment is not always helpful and may be required when cytological aspects appear unclear, when extensive fibrosis and/or hemorrhages are present and von Brunn's nests and buds are poorly visible or deep in the lamina propria. Thus, markers of cellular proliferation as Ki67 (MIB-1) could be useful to differentiate Brunn's nests or more hyperplastic nests (florid von Brunn's nests) from transitional cell carcinoma. In case 1, immunoreactivity for CK20 was mild (too long time of the sample fixation?), if compared with a clear and intense positivity of umbrella cells in the normal ureter of the case control and in human ureter and urinary bladder.

\section{Conclusion}

Von Brunn's nests of the reported cases were discovered in left ureters of cats. Ureters of humans and animals are usually not involved in these rare benign conditions. They must be differentiated from ureteral malignant neoplasms, which also represent a further uncommon disorder both in cats and other domestic animals. Histology after surgery is the main choice for diagnosis and future treatment, associated to immunohistochemical assessment only in case of difficult interpretation. In humans they are considered to be preneoplastic lesions [4] [5]. It is not excluded that von Brunn's nests are preneoplastic/premalignant lesions in animals, but this hypothesis has yet to be confirmed [3] [4] [6].

\section{Acknowledgements}

The authors thank the interpreter/translator Elvira Calabrese for revision of the text.

\section{Author Note}

This paper was presented at the 2016 34th ESVP (European Society of Veterinary Pathology) Meeting and 27th ECVP (European College of Veterinary Pathologists) Meeting, Bologna (Italy), September $7^{\text {th }}-10^{\text {th }}$ and published as abstract in Journal of Comparative Pathology, Volume 156, Issue 1, January 2017, page 88 , https://doi.org/10.1016/j.jcpa.2016.11.109.

\section{Statement}

This study is a case report carried out on archived tissue samples and histological sections of cats with ureteral lesions. The research did not influence any therapeutic decision. For this reason, approval by an Ethics Committee was not required. However, all diagnostic and therapeutic procedures were performed in accordance with current standards of animal and human care and the examined samples were routinely collected for standard diagnostic purposes.

\section{Conflict of Interest}

The authors declare no conflict of interests with respect to the publication of this article. 


\section{References}

[1] Young, R.H. (2009) Tumor-Like Lesion of the Urinary Bladder. Modern Pathology, 22, S37-S52. https://doi.org/10.1038/modpathol.2008.201

[2] Bresheas, M.A. and Confer, A.W. (2017) The Urinary System. In: Zachary, J.F., Ed., Pathologic Basis of Veterinary Disease, 6th Edition, Elsevier, St. Louis, 617-681. https://doi.org/10.1016/B978-0-323-35775-3.00011-4

[3] Cianciolo, R.E. and Mohr, F.C. (2016) Urinary System. In: Maxie, M.G., Ed., Jubb, Kennedy, and Palmer's Pathology of Domestic Animals, 6th Edition, Vol. 2, Elsevier, St. Louis, 449. https://doi.org/10.1016/B978-0-7020-5318-4.00010-3

[4] Meuten, D.J. and Meuten, T.L.K. (2017) Tumors of the Urinary System. In: Meuten, D.J., Ed., Tumors in Domestic Animals, 5th Edition, John Wiley \& Sons Inc., Ames, 687.

[5] Guo, A., Liu, A. and Teng, X. (2016) The Pathology of Urinary Bladder Lesions with an Inverted Growth Pattern. Chinese Journal of Cancer Research, 28, 107-121. http://doi.org/10.3978/j.issn.1000-9604.2016.02.01

[6] Zachary, J.F. (1981) Cystitis Cystica, Cystitis Glandularis, and Brunn's Nests in a Feline Urinary Bladder. Veterinary Pathology, 18, 113-116. https://doi.org/10.1177/030098588101800112

[7] Gelberg, H.B. (2010) Urinary Bladder Mass in a Dog. Veterinary Pathology, 47, 181-184. https://doi.org/10.1177/0300985809353176

[8] Meuten, D.J., Everitt, J., Inskeep, W., Jacobs, R.M., Peleteiro, M. and Thompson, K.G. (2004) Histological Classification of Tumors of the Urinary System of Domestic Animals. Second Series, Vol. XI, The Armed Forces Institute of Pathology, Washington DC, 25-26 and 36-38. 